\title{
Editorial
}

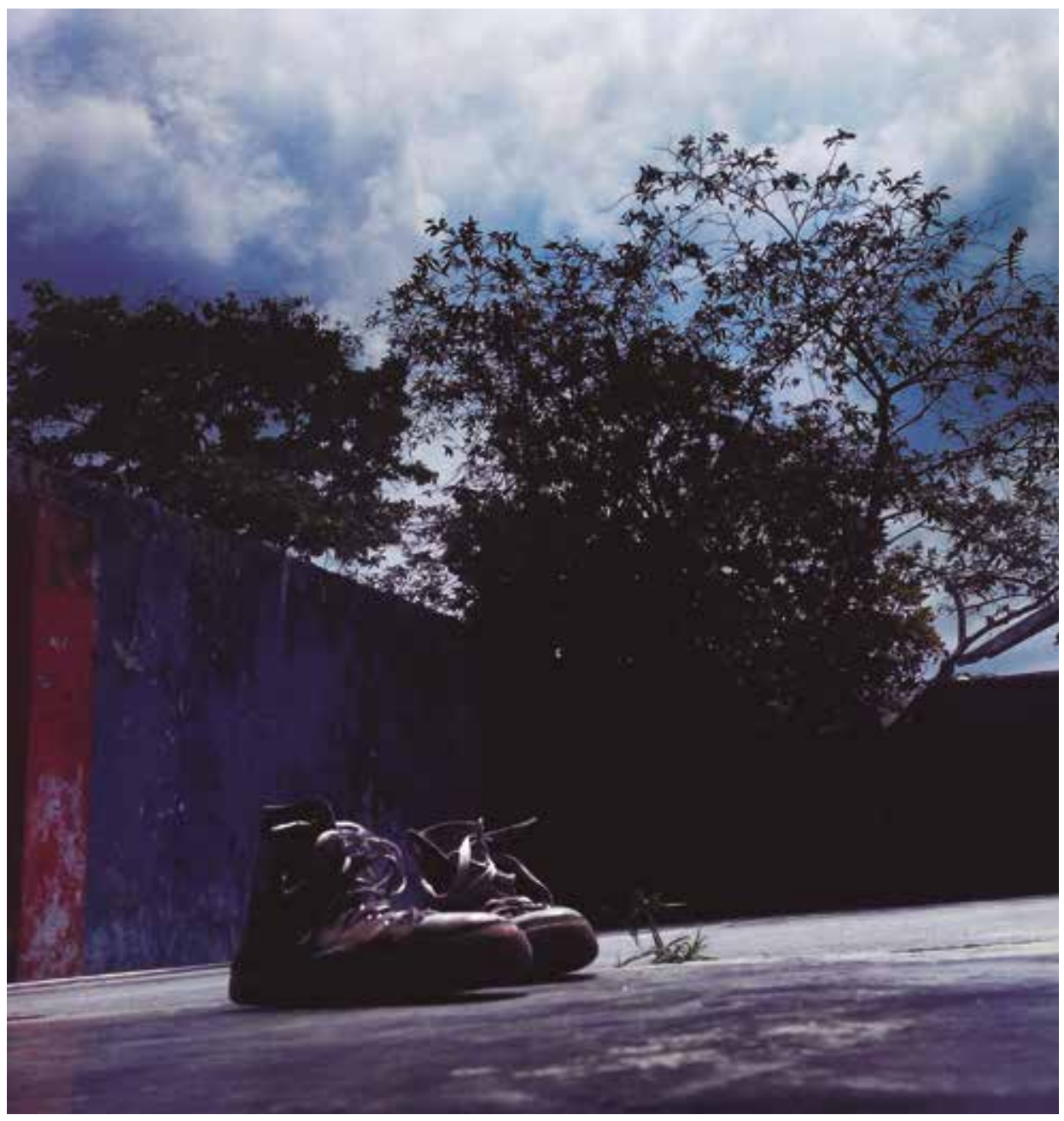

Sin título

De la serie Ungidos (o como derivar entre las rutas del agua)

Fotografía digital

2018

Medellín 


\section{Metamorfosis de la revista científica. De impresa a electrónica}

Las revistas académicas son el medio más utilizado por las universidades para divulgar y difundir su producción científica. En ellas, sus miembros presentan a las comunidades académicas y a la sociedad en general los resultados de investigación, visibilizan su producción académica y procuran generar impacto social y científico. Así, las revistas académicas y sus artículos se han convertido en los medios más expedito y efectivo para socializar la investigación académica y ponerla a disposición de la sociedad para su aprovechamiento (Aguado, Rogel, Garduño y Zúñiga, 2008a, pp. 14-15).

Desde la aparición de las primeras revistas, en la segunda mitad del siglo XVII, han sido el medio por excelencia para la publicación, difusión y divulgación del conocimiento científico de vanguardia (Novelle, 2012, pp. 39-40). No obstante, en las últimas décadas estas han sufrido una metamorfosis, pasando del tradicional formato impreso al electrónico (pp. 74-75). Los avances tecnológicos en la informática, desde la década de 1980, han traído consigo el extendido uso del computador personal, el desarrollo de Internet y la más reciente explosión de los dispositivos móviles, provocando a su vez el incremento del volumen de información y la versatilidad en su acceso y uso. Los textos digitales han ganado espacio y presencia en la cultura académica, de allí que la metamorfosis de las revistas debe verse como una adaptación de la comunidad científica y del mundo editorial a las nuevas dinámicas de la edición académica (Barrueco, García y Gimeno, 1996; Aguado, Rogel, Garduño y Zúñiga, 2008a, pp. 13-14).

Las universidades de países con niveles de desarrollo tecnológico avanzado, principalmente norteamericanas, dieron un paso agigantado al formato digital, impulsados indudablemente por las posibilidades de Internet para la disposición, almacenamiento y acceso a una enorme cantidad de textos y datos desde cualquier lugar del mundo. De esta forma en la actualidad, la mayoría de los miembros de la comunidad académica internacional publican y consultan los avances en sus respectivas áreas del conocimiento a través de artículos de revista en formato electrónico dispuestos en la World Wide Web (Fernández, Soto, Pelekais y Pelekais, 2016). 
En las tres décadas que lleva la experiencia, las revistas electrónicas han manifestado sus ventajas. El formato impreso de las revistas ha demostrado ser muy limitado para los estándares actuales, e incluso las bibliotecas están reduciendo significativamente el inventario de este tipo de publicaciones y reemplazándolo por enlaces a través de sus catálogos en línea. Lo mismo pasa con el canje entre instituciones, alegando los mismos motivos que las bibliotecas: falta de espacio y disponibilidad en Internet (Aguado, Rogel, Garduño y Zúñiga, 2008b).

La capacidad de acceso desde cualquier punto de red alrededor del mundo supera con creces las limitaciones del formato impreso, liberando recursos destinados a la impresión, distribución y almacenamiento, y llegando a una cantidad de lectores mucho mayor (Suber, 2015, p. 108). La versatilidad de los formatos permite la lectura, descarga y almacenamiento en diferentes dispositivos y en diferentes idiomas. El procesamiento de los textos permite la marcación de metadatos que facilitan la búsqueda y el acopio en repositorios y la cosecha automática. La individualización de los textos permite compartirlos fácilmente a públicos objetivos por correo electrónico y redes sociales, entre otros.

Pero tal vez una de las mayores ventajas es que se le devuelve al editor el control de sus publicaciones, cuya visibilidad estaba siendo controlada por los sistemas de indexación y resumen (SIR) de corriente principal: Web of Science y Scopus (Aparicio, Banzato y Liberatore, 2016, p. 38). Los sistemas de gestión editorial como Open Journal System (OJS) permiten el procesamiento y la publicación de los artículos, aprovechando todas las ventajas del formato electrónico frente al impreso, a lo que se suma la interoperatividad con otras plataformas que favorecen la visibilidad, como Web of Sciences y Scopus —paradójicamente-, SciELO, Redalyc, DOAJ, Dialnet, entre muchas otras.

La edición digital flexibiliza los tiempos de publicación, facilita la presentación de los textos en sus diferentes instancias — pre-print, postprint - , favoreciendo la visibilidad al publicar con mayor rapidez y fluidez los artículos en un medio que permite la difusión y la divulgación internacional de manera inmediata y simultánea (Suber, 2015, pp. 171-174). La publicación de los artículos una vez estén listos y no tener que esperar hasta la publicación completa del número es un aspecto muy bien visto por los SIR, y para las revistas y los autores implica mayor visibilidad, prestigio e impacto social y académico (pp. 196-198). 
Ciertamente, el movimiento de Acceso Abierto (Suber, 2015) ha contribuido notablemente al auge de las revistas electrónicas en Latinoamérica, al punto que es considerada como la región que más ha acogido las políticas de acceso abierto en el mundo y, por ende, la creación de revistas electrónicas o el tránsito de las tradicionales impresas a su digitalización. Colombia no ha sido la excepción y sus políticas de ciencia y tecnología (Colciencias, 2016) han proporcionado un escenario para el crecimiento exponencial de este tipo de publicaciones en el país que, al igual del resto de la región, su financiación proviene de recursos públicos, de ahí la necesidad de el acceso abierto no comercial al conocimiento (Aguado y Vargas, 2016; Fischman y Alperin, 2015).

Tras una revisión rápida por algunas bases de datos de revistas, con el objetivo de diagnosticar la metamorfosis de las revistas científicas se puede observar la siguiente situación a enero de 2019. Al consultar el portal de portales Latindex, de las 26536 revistas en su directorio, 8645 están registradas como revistas electrónicas, 254 de ellas son de Colombia. En cuanto a la plataforma de SciELO, en Colombia aparecen 226 revistas vigentes de las 1285 de toda la colección. En el caso de Redalyc, de las 1297 revistas científicas 212 son de Colombia. Por su parte, de las 12485 revistas del Directorio de Revistas de Acceso Abierto (DOAJ), 321 son revistas colombianas. Estos números son significativos ya que se trata de plataformas que exigen acceso completo en línea a los artículos. En este punto es importante señalar que incluso Web of Science y Scopus están contemplando las revistas de acceso abierto y llevando registro de ellas en su información de clasificación.

Teniendo todo esto en cuenta, desde 2010 la revista Estudios Políticos ha preparado el terreno para hacer el tránsito a la virtualidad, lo que inició con la completa gestión editorial a través de la plataforma OJS, que contempla la recepción de artículos, su evaluación y posterior publicación. Desde la edición 39 se dispone de los artículos en formato HTML y desde la edición 50 se incluye el formato XML; adicionalmente, todas las ediciones se encuentran disponibles en PDF.

Como muestra de la disponibilidad y acceso de nuestros contenidos de forma libre y gratuita, la revista se suma al movimiento de Acceso Abierto y acoge sus políticas a través de la licencia Creative Commons. Además, la revista procura un registro estricto de sus metadatos y dispone del protocolo OAI-PMH para la interoperatividad y cosecha de metadatos. En la misma 
vía, desde 2017 la página puede ser navegada con dispositivos móviles y su presencia en redes sociales contribuye notablemente a la visibilidad.

Conscientes de las nuevas condiciones de la edición científica y convencidos de que contribuirán notablemente a la calidad de la revista y a su visibilidad e impacto social y académico, Estudios Políticos ha decidido aumentar su periodicidad de semestral a cuatrimestral y enfocarse exclusivamente en la versión electrónica, con lo que se espera aumentar el flujo anual de artículos, evitar su envejecimiento y obsolescencia, y garantizar su procesamiento y publicación oportunos. Estas medidas se suman a las iniciadas en 2017 con la publicación de ediciones preliminares con artículos ya evaluados y en versión final en HTML, completamente disponibles para la lectura.

Queremos dejar claro que estas medidas no se deben a una claudicación ciega ante un modelo exótico o a una moda, todo ha sido largamente meditado y consultado con los comités Científico y Editorial, llenos de confianza en nuestra propuesta editorial, la cual debe llegar a la mayor cantidad de público en todo el mundo. No se puede pasar por alto que, sumado al componente tecnológico, la revista realiza un estricto proceso editorial que contempla, entre otros criterios internacionales, la revisión por pares doble-ciego, muestra de nuestro compromiso con la calidad editorial y científica, y con la política editorial que nos ha guiado desde 1992, la cual es difundir y divulgar artículos que brinden elementos para la explicación de la problemática política contemporánea en Colombia, América Latina y otras partes del mundo.

\section{Germán Darío Valencia Agudelo (Colombia)* Carlos Esteban Flórez Mazo (Colombia)**}

\footnotetext{
* Director revista Estudios Políticos. Economista. Especialista en Gerencia Social. Magíster en Ciencia Política. Doctor en Estudios Políticos. Grupo Hegemonía, guerras y conflictos, Instituto de Estudios Políticos, Universidad de Antioquia UdeA. Calle 70 No. 52-21, Medellín, Colombia. Correo electrónico: german.valencia@udea.edu.co - Orcid: http://orcid.org/0000-0002-6412-6986 - Google Scholar: https://scholar.google.es/citations?user=7Sm8z3MAAAAJ\&hl=es

** Editor revista Estudios Políticos. Comunicador Social-Periodista. Especialista en Edición de Publicaciones. Instituto de Estudios Políticos, Universidad de Antioquia UdeA. Calle 70 No. 52-21, Medellín, Colombia. Correo electrónico: esteban.florez@udea.edu.co

Cómo citar este artículo: Valencia Agudelo, Germán Darío y Flórez Mazo, Carlos Esteban. (2019). Editorial. Metamorfosis de la revista científica. De impresa a electrónica. Estudios Políticos (Universidad de Antioquia), 54, pp. 9-13. http://doi.org/10.17533/udea.espo.n54a01
} 


\section{Referencias bibliográficas}

1. Aguado López, Eduardo; Rogel Salazar, Rosario; Garduño Oropeza, Gustavo; Zúñiga, María Fernanda. (2008a). Redalyc: una alternativa a las asimetrías en la distribución del conocimiento científico. Ciencia, Docencia y Tecnología, xIx (37), pp. 11-30.

2. Aguado-López, Eduardo; Rogel-Salazar, Rosario; Garduño-Oropeza, Gustavo; Zúñiga, María Fernanda. (2008b). Cambios de la comunicación científica Iberoamericana, el caso de Redalyc y su contribución a la visibilidad de las revistas especializadas. Quórum. Revista de Pensamiento Iberoamericano, 22, pp. 149-168.

3. Aguado-López, Eduardo; Vargas Arbeláez, Esther Juliana. (2016). Reapropiación del conocimiento y descolonización: el acceso abierto como proceso de acción política del sur. Revista Colombiana de Sociología, 39 (2), pp. 69-88.

4. Aparicio, Alicia; Banzato, Guillermo y Liberatore, Gustavo. (2016). Manual de gestión editorial de revistas científicas de ciencias sociales y humanas: buenas prácticas y criterios de calidad. Buenos Aires: Pisac. Recuperado de http://www. mincyt.gob.ar/_post/descargar.php?idAdjuntoArchivo $=50768$

5. Barrueco Cruz, José Manuel; García Testal, Cristina y Gimeno, María José. (1996). Una aproximación a las revistas científicas en formato electrónico. Revista Española de Documentación Científica, 19 (3), pp. 304-313.

6. Departamento Administrativo de Ciencia, Tecnología e Innovación (Colciencias). (2016). Política nacional para mejorar el impacto de las publicaciones científicas nacionales. Versión final. Bogotá, D. C.: Colciencias. Recuperado de http://colciencias.gov.co/sites/default/files/upload/noticias/120816-vfpolitica_ publindex 2.0 og ao miv.pdf

7. Fernández de Pelekais, Cira Arelis; Soto, Maricarmen; Pelekais, Elmar Aldrin y Pelekais, Elmars Andris. (2016). De la publicación impresa a la electrónica: una simbiosis de elementos para divulgar la producción científica en las organizaciones universitarias. Paakat: Revista de Tecnología y Sociedad, 6 (10). Recuperado de http:// www.udgvirtual.udg.mx/paakat/index.php/paakat/article/view/266/html

8. Fischman, Gustavo y Alperin, Juan Pablo. (2015). Sobre luces y sombras. Las revistas científicas hechas en Latinoamérica. En: Alperin, Juan Pablo y Fischman, Gustavo (eds.). Hecho en Latinoamérica. Acceso abierto, revistas académicas e innovaciones regionales (pp. 13-17). Buenos Aires: Clacso. Recuperado de http:// biblioteca.clacso.edu.ar/clacso/se/20150722110704/HechoEnLatinoamerica.pdf

9. Novelle López, Laura. (2012). De la arcilla al E-book. Historia del libro y las bibliotecas. Recuperado de http://eprints.rclis.org/17420/1/NOVELLE\%20 L\%C3\%93PEZ, \%20LAURA \% 20-\%20De\%20la\%20arcilla\%20al\%20E-book.pdf

10. Suber, Peter. (2015). Acceso abierto. Toluca: UAEM. Recuperado de http:// biblioteca.clacso.edu.ar/clacso/otros/20150820022027/PeterSuber.pdf 